\title{
Successful Percutaneous Closure of Left Atrial Appendage with Periprocedural Hemorrhage Stopped by the Implanted Device
}

\author{
Maksym J, Mazurek T*, Kochman J, Grygier M, Kapłon-Cieślicka A, Marchel M, Lodziński P, Wilimski R, \\ Czub P, Hendzel P and Opolski G
}

Chair and Department of Cardiology, Medical University of Warsaw, Poland

Received: April 25, 2018; Published: May 09, 2018

*Corresponding author: Tomasz Mazurek, Chair and Department of Cardiology, Medical University of Warsaw, ul. Banacha 1a, 02-097 Warsaw, Poland

Abstract

Atrial fibrillation (AF) is the most common arrhythmia and affects $1 \%$ of the population. It is associated with the need for oral anticoagulation therapy for stroke prevention. Based on the fact that more than $90 \%$ of stroke causing thrombus has its origin within the left atrial appendage (LAA) [1], nonpharmacological approaches such as isolation of LAA from the blood circulation were proposed. However, this invasive way of stroke prevention has its own risk related to the device and procedure.

Keywords: Atrial Fibrillation; Left Atrial Appendage Closure; Pericardial Effusion

Abbreviations: AF: Atrial Fibrillation; NVAF: Non-Valvular Atrial Fibrillation; LAA: Left Atrial Appendage; LAAC: Left Atrial Appendage Closure; NYHA: New York Heat Association; TTE: Transthoracic Echocardiography; TEE: Transesophageal Echocardiography; INR: International Normalized Ratio; TEE: Transesophageal Echocardiography; IAS: Interatrial Septum; UFH: Un Fractionated Heparin; RV: Right Ventricle; ACT: Activated Clotting Time3. Abbreviations: AF: Atrial Fibrillation; NVAF: Non-Valvular Atrial Fibrillation; LAA: Left Atrial Appendage; LAAC: Left Atrial Appendage Closure; NYHA: New York Heat Association; TTE: Transthoracic Echocardiography; TEE: Transesophageal Echocardiography; INR: International Normalized Ratio; TEE: Transesophageal Echocardiography; IAS: Interatrial Septum; UFH: Un Fractionated Heparin; RV: Right Ventricle; ACT: Activated Clotting Time

\section{Introduction}

Atrial fibrillation (AF) is the most common cardiac arrhythmia affecting $1 \%$ of the population and is associated with fourfold to fivefold increase in the risk of ischemic stroke, regardless of etiology [2-5]. In recent years the left atrial appendage (LAA) has received significant medical evidence for its role in generating strokecausing blood clots in patients with non-valvular atrial fibrillation (NVAF). Left atrial appendage closure (LAAC) with percutaneously implanted occluder in the LAA has been shown to be non-inferior to warfarin in the prevention of ischemic stroke in patients with NVAF [5]. This way of stroke prevention may be considered in patients with high bleeding risk, who have a history of serious bleeding complication and because of which have absolute contraindication to oral anticoagulation (OAC) therapy. The Watchman device (Boston Scientific Corp., Marlborough, MA, USA) was the first of its kind alternative to OAC therapy and its safety and effectiveness was demonstrated in randomized, clinical trials [6-8]. Nonetheless, the LAAC procedure is associated with complications related to the device, such as pericardial effusion, device embolization, and device-related thrombus formation [6-9].

\section{Case Report}

A 61-year-old man with paroxysmal NVAF and several comorbidities including hypertension, coronary artery disease, heart failure in NYHA class II and hyperlipidemia was admitted to the hospital for the assessment of indications and feasibility of the percutaneous LAAC. Both thromboembolic and bleeding risk were high as demonstrated by the CHA2DS2-VASc and HAS-BLED scores, with 3 points of each. Furthermore patient had an absolute contraindication to OAC therapy due to reported inability to maintain the international normalized ratio (INR) within the therapeutic range, and due to the repeated history of severe gastrointestinal bleeding during vitamin OAC therapy. In order to the qualification to the procedure the transesophageal echocardiography (TEE) was performed to assess the anatomy of LAA: depth, shape and width 
of the orifice. Evaluation of the interatrial septum (IAS) before its puncture and exclusion of the presence of thrombi inside the LAA were also performed during TEE.

Based on the patient's history, high bleeding and thromboembolic risk and good LAA morphology for the procedure the patient was qualified for the percutaneous LAAC. The procedure was performed under general anesthesia with TEE and fluoroscopic guidance. After trans septal puncture un fractionated heparin (UFH) was administrated to continue the procedure with prolonged activated clotting time (ACT) to at least $250 \mathrm{~s}$. Following the sheath introduction to the LAA, angiographic projections were made to assess the shape of LAA. Based on acquired angiographic planes and TEE visualization, the $24 \mathrm{~mm}$ Watchman device has been chosen. During the procedure of positioning the device, fluid appeared in the pericardial sac, what was observed on TEE (Figure 1).

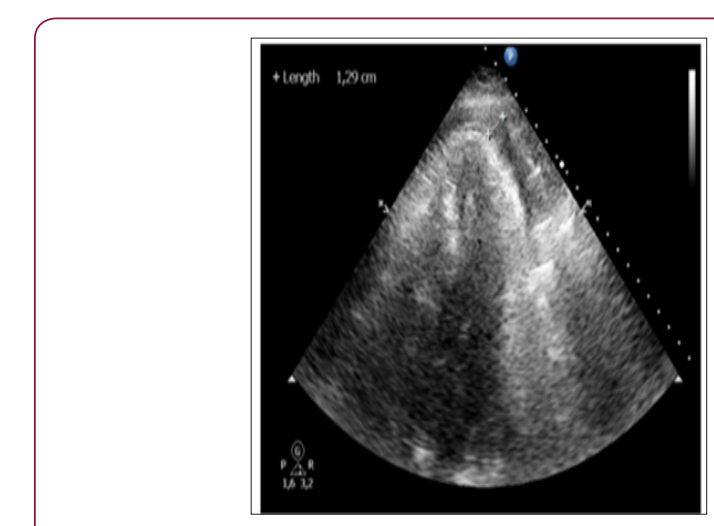

Figure 1: Pericardial effusion in TEE.

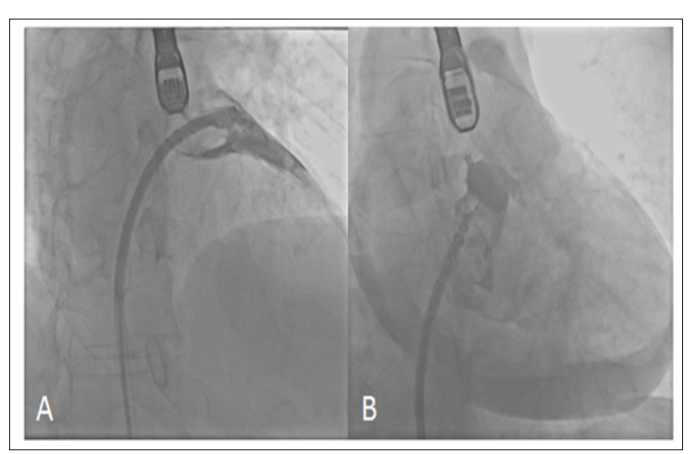

Figure 2: Pericardial effusion in fluoroscopy.

In fluoroscopy it was evident that contrast is getting through the LAA to pericardium, thus the injury to the LAA wall was confirmed (Figure 2). In TEE the pericardial effusion was enlarging during the procedure up to $16 \mathrm{~mm}$ behind the right ventricle (RV). At this moment the heart rate kept stable on the level of 60 beats per minute, but the drop of systolic blood pressure to $60 \mathrm{mmHg}$ was observed. Because of the patient's hemodynamic instability, the intravenous adrenaline and saline infusion was started. The procedure was completed with successful device deployment with no leaks in TEE evaluation. Moreover, thanks to its optimal position in the LAA, that excluded LAA from blood circulation, there was no increase of pericardial effusion. After completing procedure patient was referred to the cardiac intensive care unit, where adrenaline infusion was continued. The next day thoracic echocardiography (TTE) has shown that the level of fluid maintains stable, with no increase in its amount and there was no compression on heart's cavities. With this TTE result it was safe to initiate the dual antiplatelet therapy to avoid the thrombus formation on the device. Repeated TTE assessments revealed decreasing amount of the fluid in pericardial sac to $4 \mathrm{~mm}$ in the ninth day after LAAC procedure. Six weeks later the follow-up TEE and TTE were performed and neither fluid in pericardial sac, nor thrombus on the device was present.

\section{Discussion}

Pericardial effusion is one of the serious adverse events related to the LAAC procedure and constituted the majority of complications. The PROTECT AF trial was the first randomized trial assessing the efficacy and safety of Watchman device [6]. The study revealed that pericardial effusion requiring surgical intervention or pericardiocentesis appeared in $1.6 \%$ and $2.4 \%$ of cases, respectively. Procedure-related pericardial effusion with tamponade was relatively frequent but not fatal [10]. The other randomized trial, that let the device get into the daily clinical practice, was PREVAIL trial. The adverse events related to the procedure was significantly lower than in PROTECT AF trial and occurred in $4.2 \%$ of the cases in Watchman arm [8]. Pericardial effusions that required to be managed with surgical intervention decreased from $1.6 \%$ to $0.4 \%$, while those required pericardiocentesis decreased from $2.4 \%$ to $1.5 \%$ [8]. The EWOLUTION registry was created to collect real-life data regarding LAAC procedure and more than thousand patients were included. The overall rate of complications during 7 days after procedure was $2.8 \%$, which is lower than in any previous conducted trial or registry [11].

Pericardial effusion appeared only in 5 cases including only one cardiac tamponade. Comparing the data previously revealed from the PROTECT AF trial and CAP (Continued Access PROTECT AF Registry) study and PREVAIL trial showed learning curve demonstrated improved implant profile in experienced and new operators $[8,10]$. The safety profile of EWOLUTION registry was also favorable in comparison to previous results. Pericardial effusion appears to be a serious complication, usually requiring management with catheter or surgical drainage. Nevertheless, patients that could be managed conservatively had no long-lasting consequences and no associated deaths have been reported after pericardial effusion in clinical trials, as well as in single center reports [12]. Our case proves that successful implantation of the device effectively eliminates LAA from the blood circulation and can prevent pericardial effusion during LAAC, which results in avoiding tamponade and the need of further interventions.

\section{Conclusion}

The safety profile of the LAAC procedures improved during last few years, even in high risk patients with several co-morbidities. The improvement in device deployment techniques and increased operator's experience decreased procedure related complications. 


\section{References}

1. Bin Kong, Yu Liu, He Huang, Hong Jiang, Congxin Huang (2015) Left atrial appendage closure for thromboembolism prevention in patients with atrial fibrillation: advances and perspectives. J Thorac Dis 7(2): 199-203.

2. Wolf PA, Abbott RD, Kannel WB (1991) Atrial fibrillation as an independent risk factor for stroke. The Framingham Study. Stroke 22: 983-988.

3. Lip G, Hoong SL (2007) Atrial Fibrillation and stroke prevention. Lancet Neurol 6: 981-993.

4. Mazurek T, Kiliszek M, Kobylecka M, Skubisz-Głuchowska J, Kochman J, et al. (2014) Relation of proinflammatory activity of epicardial adipose tissue to the occurrence of atrial fibrillation. Am J Cardiol 113(9): 15051508.

5. Mazurek T, Kobylecka M, Zielenkiewicz M, Kurek A, Kochman J, et al. (2017) PET/CT evaluation of ${ }^{18}$ F-FDG uptake in pericoronary adipose tissue in patients with stable coronary artery disease: Independent predictor of atherosclerotic lesions' formation? J Nucl Cardiol 24(3): 1075-1084.

6. Holmes DR, Reddy VY, Turi ZG, Doshi SK, Sievert H, et al. (2009) Percutaneous closure of left atrial appendage versus warfarin therapy for prevention of stroke in patients with atrial fibrillation: a randomized non-inferiority trial. Lancet 374: 534-542.

7. Reddy VY, Doshi SK, Sievert H, Buchbinder R, Neuzil P, et al. (2013) Percutaneous Left Atrial Appendage Closure for Stroke Prophylaxis in
Patients with Atrial Fibrillation. 2.3-Year Follow-up of the PROTECT AF. Circulation 127: 720-729.

8. Holmes DR, Kar S, Price MJ, Whisenant B, Sievert H, et al. (2014) Prospective Randomized Evaluation of the Watchman Left Atrial Appendage Closure Device in Patients with Atrial Fibrillation Versus Long-Term Warfarin Therapy: the PREVAIL trial. J Am Coll Cardiol 64(11): 1186

9. Mobius Winkler S, Majunke N, Sandri M, Mangner M, Linke A, et al. (2015) Percutaneous left atrial appendage closure: Technical aspects and prevention of periprocedural complications with the watchman device. World J Cardiol 7: 65-75.

10. Reddy VY, Holmes D, Doshi SK, Neuzil P, Kar S (2011) Safety of percutaneous left atrial appendage closure: results from the Watchman Left Atrial Appendage System for Embolic Protection in Patients with AF (PROTECT AF) clinical trial and the Continued Access Registry. Circulation 123(4): 417-424.

11. Boersma L, Schmidt B, Betts TR, Sievert H, Tamburino C, et al. (2016) Implant success and safety of left atrial appendage closure with the WATCHMAN device: peri-procedural outcomes from the EWOLUTION registry. Eur Heart J 37(31): 2465-2474.

12. Maksym J, Mazurek T, Kochman J, Grygier M, Kapłon-Cieślicka A, et al. (2018) Dual antiplatelet therapy is safe and efficient after left atrial appendage closure. Kardiol Pol 76(2): 459-463.

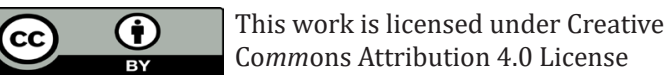

Submission Link: https://biomedres.us/submit-manuscript.php

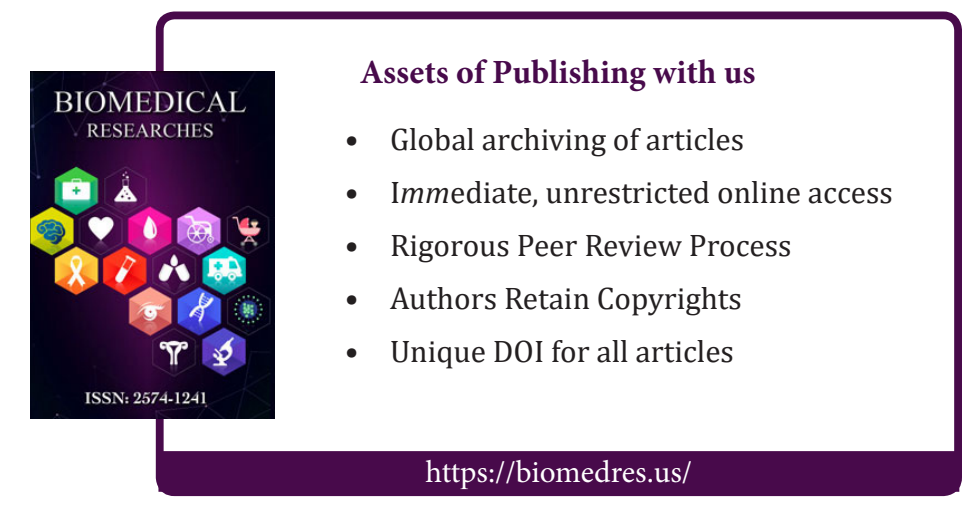

